\title{
Chemiresistiver Toluolsensor auf Basis von Polymerkompositen
}

\author{
Maryam Ehsani, Frederic Güth und Yvonne Joseph \\ Institut für Elektronik- und Sensormaterialien, TU Bergakademie Freiberg, Freiberg, Deutschland \\ kontakt: maryam.ehsani@doktorand.tu-freiberg.de
}

\section{Einleitung}

Die Überwachung von flüchtigen organischen Verbindungen (VOC) in der Luft ist heute in vielen Ländern aufgrund ihrer gesundheitlichen Auswirkungen und der geltenden Vorschriften ein wichtiges Forschungsthema. Toluol ist eine der wichtigsten flüchtigen organischen Verbindungen, die zu den Schadstoffemissionen bei der Herstellung von Benzin und anderen Kraftstoffen beitragen. Für die Erkennung von toxischen Gasen wurden viele Arten von Sensoren entwickelt, die verschiedene Sensormaterialien wie z.B. Metalloxide verwenden. Der größte Nachteil dieser Materialien ist, dass sie meist hohe Arbeitstemperaturen erfordern $[1,2]$. Daher ist die Entwicklung von Sensormaterialien, die bei Raumtemperatur arbeiten können, wichtig für die Entwicklung von Sensoren, die ein einfaches Design, geringere Kosten sowie eine höhere Empfindlichkeit und Effizienz aufweisen [2]. Nanokomposit-Materialien auf Polymerbasis sind in letzter Zeit für sensorische Anwendungen von großem Interesse. Ein wichtiges Problem, das einer erfolgreichen Sensorleistung im Wege steht, ist jedoch häufig die mangelnde elektrische Leitfähigkeit. Auf Graphen ( $G$ ) basierende Materialien werden aufgrund ihrer außergewöhnlichen chemischen, physikalischen und molekularen Eigenschaften häufig zur Verbesserung der elektrischen Leitfähigkeit von polymerbasierten Gassensoren eingesetzt [35]. Die hohe chemische Aktivität und die hohe Elektronenübertragungsfähigkeit von Nanomaterialien auf Graphenbasis sind auf die große Oberfläche und das hohe Oberflächen-Volumen-Verhältnis des Materials zurückzuführen [68]. Graphenoxid (GO) und reduziertes Graphenoxid (rGO) sind vielversprechende Nanomaterialien für die Herstellung von Gassensoren aufgrund ihrer sauerstoffhaltigen Gruppen wie Epoxid, Carboxyl, Carbonyl und Hydroxyl, die das Material funktionalisierbar machen $[9,10]$. Im Vergleich zu GO enthält rGO weniger solche Gruppen und zeigt aufgrund der Hydrophobie der Oberfläche eine stabilere Dispersion in organischen Lösungsmitteln, was zu einer besseren Verteilung in der Matrix und damit zu einer höheren elektrischen Leitfähigkeit führt. rGO kann für elektronische Geräte, Nanokomposit-Materialien, Polymerkomposit-Materialien, biomedizinische Anwendungen, Katalyse und vieles mehr verwendet werden [11,12]. Für Sensoranwendungen sind die Eigenschaften von Nanokomposit-Sensoren auf der Basis von rGO/Polymermaterial jedoch noch unbefriedigend [13-17], weshalb versucht wird die Eigenschaften durch weitere Modifikationen zu verbessern. In dieser Arbeit wurde Tetraethylenpentamin (TEPA) zur Reduktion und Funktionalisierung von GO (F-rGO) verwendet, um die
Dispersionsqualität von rGO in Polymeren (Polymethylmethacrylat, PMMA) zu optimieren. TEPA enthält eine große Anzahl von Amingruppen, die die funktionellen Sauerstoffgruppen von $\mathrm{GO}$ entfernen und somit zu einer höheren Defektdichte führen, was die elektrische Leitfähigkeit des Komposits erhöhen kann (Abb.1). Das fertige FrGO/Polymer-Komposit kann als sensitive Schicht zum Nachweis unterschiedlicher Konzentrationen von Toluoldämpfen eingesetzt werden.

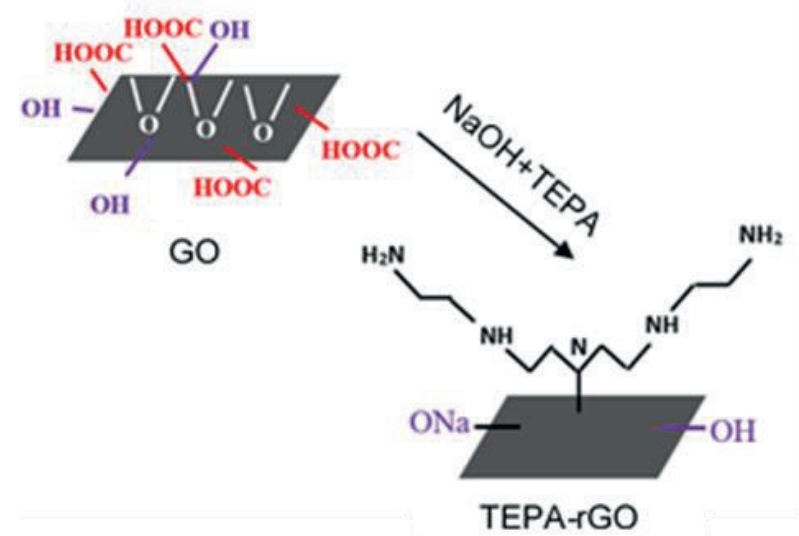

Abb. 1: Illustration der Reaktivität von $\mathrm{GO}$ bei $\mathrm{NaOH}$ und TEPA-Behandlung

\section{Methoden und Materialien}

Als Polymerkomponente der hier präsentierten Komposite wurde Polymethylmethacrylat (PMMA) gewählt, welches mittels Emulsionspolymerisation synthetisiert wurde. Für die Polymersynthese wurde das Monomer (MMA) als Lösung $(20 \mathrm{ml}, 99 \%$, Alfa Aesar) zu deionisiertem Wasser $(200 \mathrm{ml})$ gegeben und unter Stickstoffspülung bei einer Temperatur von $60{ }^{\circ} \mathrm{C}$ auf einer Heizplatte gerührt (350 U/min, 1:30 h). Die Stickstoffspülung diente dazu, den Sauerstoff aus der Reaktionsumgebung zu entfernen.

Weiterhin wurde Azobis-(2-Methylpropionamidin) Dihydrochlorid $(0,10 \mathrm{~g}$, Fluka) als Radikalinitiator mit deionisiertem Wasser (50 ml) vermischt, bis eine homogene Lösung entstand. Dieser Radikalstarter fördert die Polymerisierung, da die sonstige Bildung von Radikalen durch Sauerstoff in der Reaktionsumgebung behindert werden kann. Danach wurde der Initiator zur MMA-Lösung hinzugefügt und die Polymerisation für 6 Stunden bei einer Temperatur von $70{ }^{\circ} \mathrm{C}$ durchgeführt. Für die Synthese von funktionalisiertem rGO (F-rGO) wurden 100 mg GO (Sigma-Aldrich) zu deionisiertem Wasser $(50 \mathrm{ml})$ hinzugefügt und 2 Stunden lang beschallt (Sonorex, super 10P). Danach wurden $2 \mathrm{ml}$ 2 molarer Natriumhydroxidlösung und $100 \mu$ I TEPA zur GO- 
Lösung gegeben und 15 Stunden lang bei Raumtemperatur mit einer Geschwindigkeit von 250 U/min gerührt. Natriumhydroxid wurde als Katalysator verwendet, um die Oberfläche von GO zu modifizieren und das Reaktionsmedium basisch zu machen. Natriumhydroxid reagiert mit den Epoxidgruppen und erzeugt eine $\mathrm{OH}$ - und eine $\mathrm{ONa}-\mathrm{Gruppe}$ auf der Oberfläche der Graphenoxidschicht (Abb.1). Dadurch wird der Ladungstransfer im F-rGO beschleunigt $[18,19]$. Schließlich wurde das F-rGO abfiltriert, mit deionisiertem Wasser gewaschen und in einem Trockenschrank bei $60{ }^{\circ} \mathrm{C}$ getrocknet. Die fertige Probe wurde anschließend in deionisiertem Wasser redispergiert und erneut im Ultraschallbad 1 Stunde lang bei einer Leistung von $10 \%$ bei Raumtemperatur beschallt, um eine stabile Dispersion herzustellen, die anschließend bei Raumtemperatur gelagert wurde. Das Nanokomposit wurde mit der Emulsionsmischmethode hergestellt. Das F-rGO wurde dem Polymer in verschiedenen Massenverhältnissen (F-rGO:Polymer 1:5, 1:1, 5:1) zugegeben und die erhaltene Lösung wurde bei einer Rotationsgeschwindigkeit von $350 \mathrm{U} / \mathrm{min}$ bei $50{ }^{\circ} \mathrm{C}$ gerührt. Das erhaltene Komposit wurden zur strukturellen und morphologischen Analyse auf ein Siliziumsubstrat getropft. Die strukturellen und morphologischen Eigenschaften der Sensorschichten wurden durch Ramanspektren und Rasterelektronenmikroskopie (REM, Carl Zeiss LEO 1530) charakterisiert. Die Dicke der Schichten wurde mittels Rasterkraftmikroskopie gemessen. Zur Sensorherstellung wurden die Nanokomposite $(0,25 \mathrm{ml}$ je Schicht, einmal wiederholt, Gesamtvolumen 0,5 ml) auf die Oberfläche einer goldenen Interdigitalelektrode (Abmessungen der empfindlichen Oberfläche: $3 \mathrm{~mm} \times 2 \mathrm{~mm}$ ) bei Raumtemperatur mit einer Geschwindigkeit von 3000 U/min für 9 Sekunden (Spincoater-BLE-Delta 10) aufgeschleudert. An diesen Schichten wurde der elektrische Widerstand mit einem Digitalmultimeter (METEX-m-3640D) bestimmt. Die sensorischen Eigenschaften wurden als Änderung des elektrischen Widerstands des Nanokompositfilms bei verschiedenen ToluolKonzentrationen (100 ppm, 400 ppm, 1600 ppm, 5000 ppm) mit einem in [20] beschriebenen Messaufbau bestimmt.

\section{Ergebnisse und Diskussion}

Ramanspektren wurden sowohl vom reinen GO, dem FrGO und den F-rGO-Polymer-Kompositen aufgenommen und sind in Abbildung 2 gezeigt. Die Spektren zeigen zwei Hauptschwingungsmoden im Bereich zwischen $1000 \mathrm{~cm}-1$ und $1750 \mathrm{~cm}-1$, die durch D- und G-Schwingungsbänder verursacht werden. Das D-Band entsteht aus einem Atmungsmodus von K-Punkt-Photonen der A1g-Symmetrie [21] und zeigt $\mathrm{sp}^{3}$-hybridisierten Kohlenstoff an. Damit zeigt eine höhere Intensität des D-Bands eine zunehmende Unterbrechung der aromatischen $\pi$-Elektronen an. Die GBande hängt mit der Streuung von E2g-Phononen erster Ordnung durch $\mathrm{sp}^{2}$-hybridisierten Kohlenstoff zusammen [10]. Das Intensitätsverhältnis (ID/ IG) von D- und G-Bindungen liefert somit Informationen über die Umwandlung von $\mathrm{sp}^{2}$-hybridisiertem Kohlenstoff in $\mathrm{sp}^{3}$-hybridisierten
Kohlenstoff und damit über die Defekte in graphenbasierten Proben, wobei ein höheres Verhältnis mehr Defekte auf den Graphenschichten anzeigt. Der Vergleich der Spektren von F-rGO: Polymer 1:5 und 5:1 zeigt, dass das Intensitätsverhältnis (ID/IG) mit der Zugabe von F-rGO steigt. Es werden also mit steigender F-rGO-Konzentration mehr Defekte erzeugt, die in einer niedrigeren elektrischen Leitfähigkeit resultieren. Das Intensitätsverhältnis der 1:1-Probe zeigt den gleichen Trend, allerdings ist die Intensität deutlich geringer, was vermutlich auf Schwierigkeiten bei der Messung zurückzuführen ist. Weiterhin kann in den Spektren ein 2DBand beobachtet werden, welches hauptsächlich etwas über die Anzahl der Schichten in der Struktur aussagt. Ein Aufspalten des Peaks, wie es für das F-rGO in Abbildung 2(a) zu erkennen ist, deutet auf das Vorliegen einer Multilagenstruktur hin. Auch die 1:1- und 5:1-Probe zeigen diese Form des 2D-Peaks und liegen somit als Multilagen vor. Abweichend davon ist im Spektrum der 1:5-Probe kein Aufspalten zu erkennen. Sie liegt somit als Monolage vor. Da dies den Ladungstransfer vereinfacht, ist zusammen mit der durch das niedrige ID/IG-Verhältnis angezeigte hohe Anzahl von delokalisierten Elektronen aufgrund des $\mathrm{sp}^{2}$ hybridisierten Kohlenstoffs für dieses Massenverhältnis von einer höheren elektrischen Leitfähigkeit auszugehen.

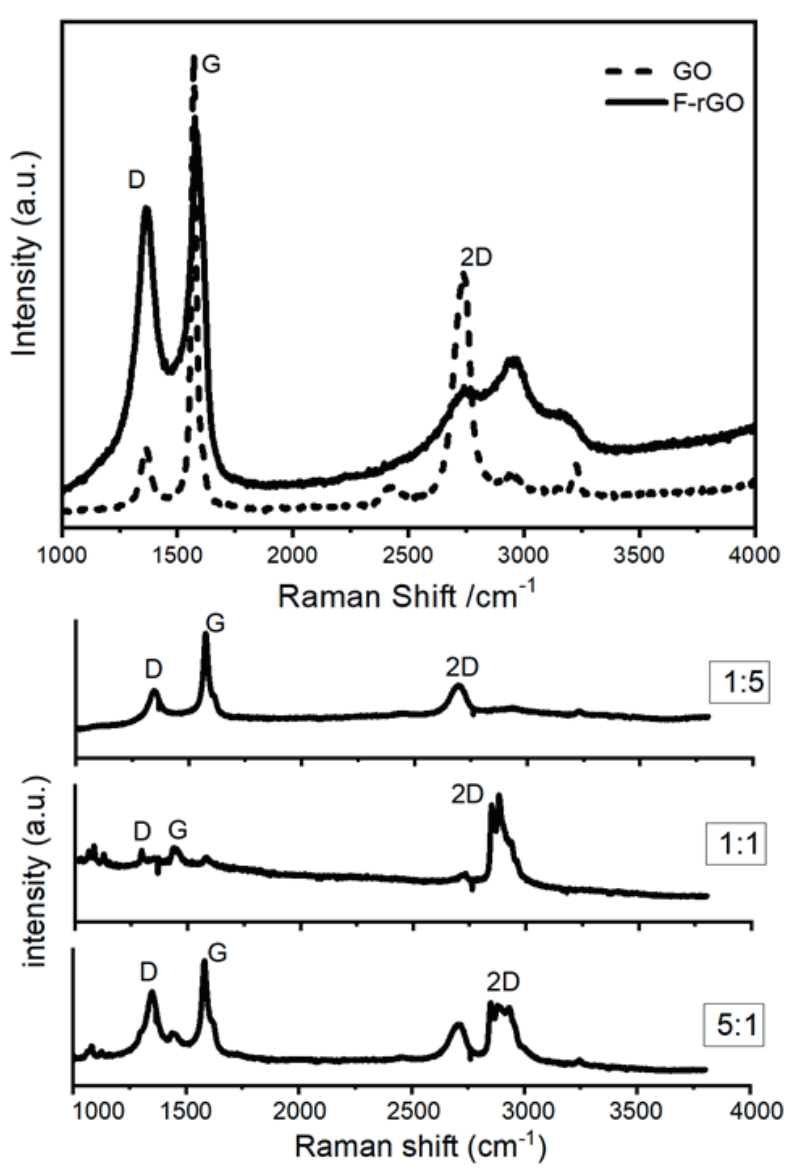

Abb. 2: Raman-Spektren von (a) GO und, F-rGO, (b) Kompositen F-rGO:Polymer (1:5, 1:1, 5:1).

In Abb. 3 (a-c) sind REM-Aufnahmen der F-rGO:Polymer-Komposite gezeigt. In diesen Bildern ist das F-rGO 
weiß und die Polymermatrix schwarz dargestellt. F-rGO/Polymer-Komposite entstehen durch elektrostatische Wechselwirkung aufgrund der zahlreichen Aminogruppen in den F-rGO-Lagen. Die Aminogruppen sorgen für eine raue, zerknitterte und faltige Oberfläche der Komposite [23]. Aufgrund der Bildung kovalenter Bindungen zwischen dem Hybridmaterial und der Polymermatrix durch die Aminogruppen wurde eine Agglomeration in den Kompositen beobachtet [24,25]. Von allen Kompositen zeigt F-rGO:Polymer 1:5 die homogenste Verteilung des F-rGO im Polymer, eine geringe Aggregation der Partikel und die Bildung eines Netzwerks in der Matrix, was zu einem vielversprechenden Kompositfilm für einen Toluolsensor führt. Die Tab. 1 zeigt die gemessene elektrische Leitfähigkeit und Dicke der Komposite.

Tab. 1: Elektrische Leitfähigkeit und Schichtdicke der Komposite.

\begin{tabular}{|l|c|c|c|}
\hline F-rGO:Polymer & $\mathbf{1 : 5}$ & $\mathbf{1 : 1}$ & $\mathbf{5 : 1}$ \\
\hline $\begin{array}{l}\text { Elektrische Leitfähigkeit } \times 10^{-7} \\
(\mathrm{~S} / \mathrm{cm})\end{array}$ & 49 & 8.7 & 8.8 \\
\hline $\begin{array}{l}\text { Dicke des Sensorfilms } \\
(\mu \mathrm{m})\end{array}$ & \multicolumn{3}{|c|}{$8 \mu \mathrm{m} \pm 0.6$} \\
\hline
\end{tabular}
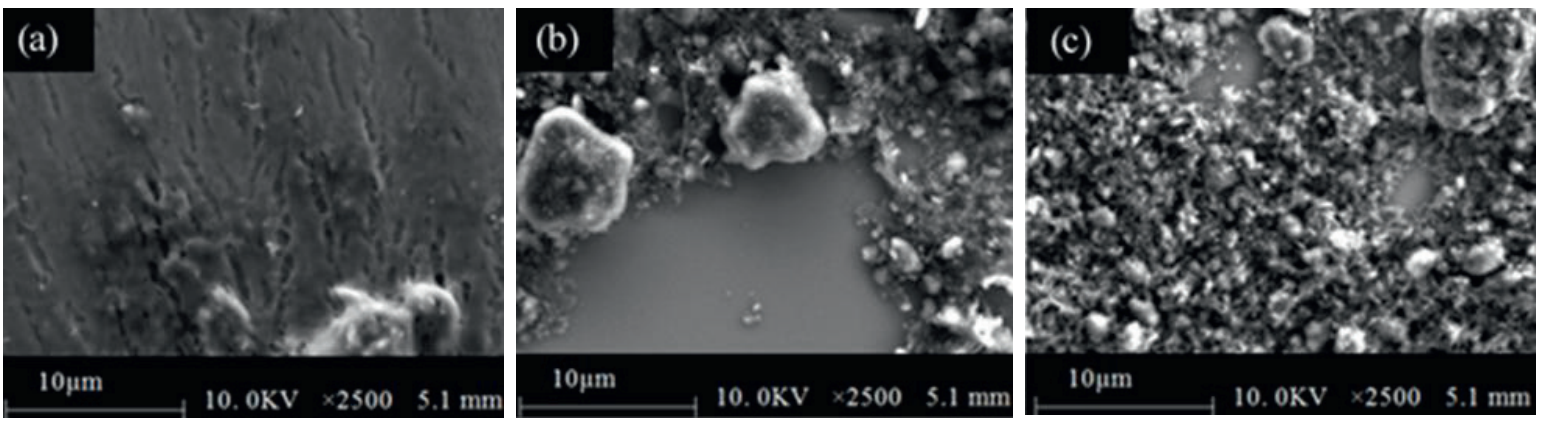

Abb. 3: REM-Bilder von F-rGO:Polymer (PMMA): (a) 1:5 (b) 1:1, (c) 5:1
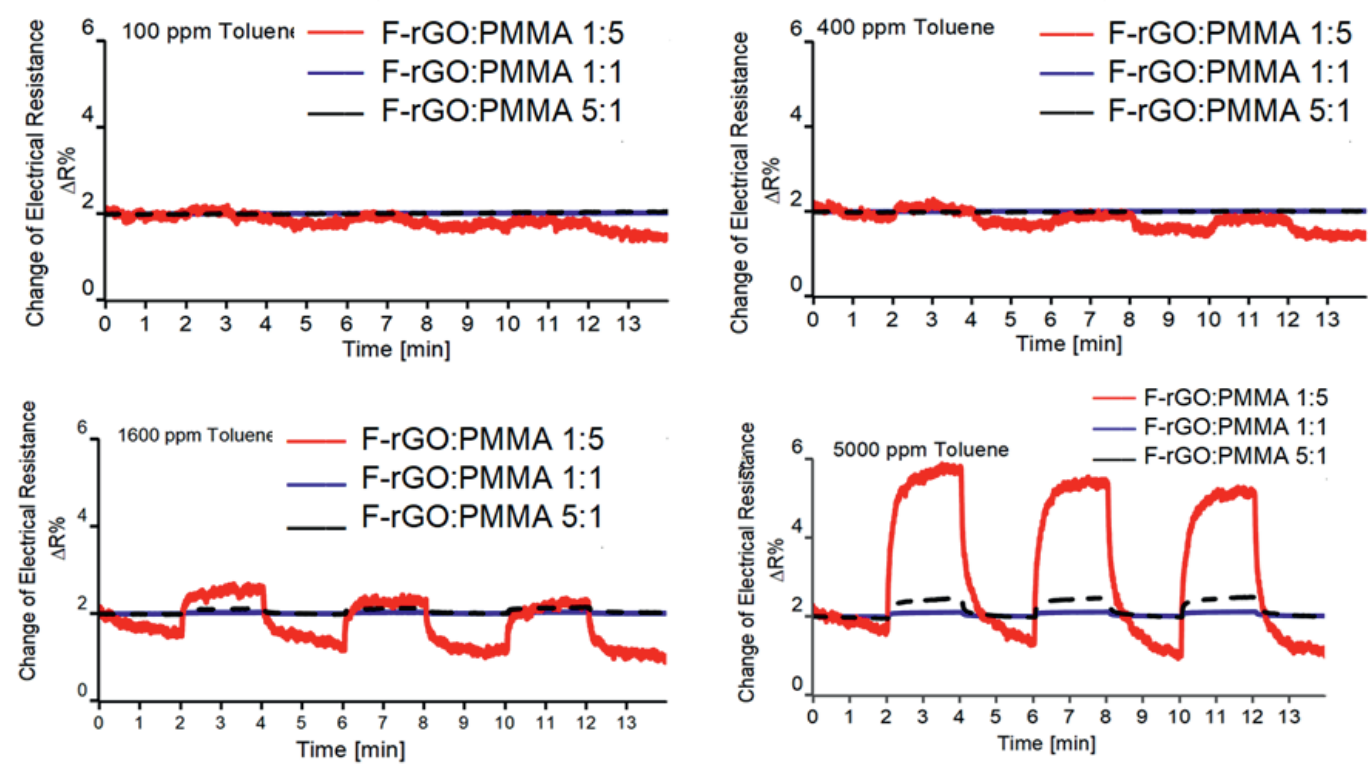

Abb. 4: Veränderung des elektrischen Widerstands von F-rGO:Polymer (PMMA) bei verschiedenen Beladungsverhältnissen von 1:5 (rote Kurve); 1:1 (blaue Kurve); und 5:1 (schwarze Kurve) gegenüber verschiedenen Konzentrationen von Toluol (100 ppm, 400 ppm, 1600 ppm und 5000 ppm). 
Der Sensor auf der Basis von F-rGO:Polymer 1:5 zeigt die höchste Empfindlichkeit. Wahrscheinlich ist dies auf die hohe elektrische Leitfähigkeit zurückzuführen, die fünfmal höher ist als bei den anderen Zusammensetzungen.

Die Widerstandsänderung von F-rGO:Polymer 1:5 wurde mit $2.17 \%$ für 100 ppm und $5.71 \%$ für 5000 ppm ermittelt. Die höhere Empfindlichkeit von F-rGO:Polymer 1:5 könnte auf die starke Bindung zwischen Polymer und F-rGO zurückzuführen sein, die $\pi-\pi$ Wechselwirkung mit dem Analyt und die homogene Dispersion des Hybridmaterials in der Matrix begünstigt. Alle untersuchten Sensoren zeigten kurze Ansprech- und Erholungszeiten, was darauf hindeutet, dass die Toluol-Moleküle im Komposit physisorbiert sind. Mit steigendem F-rGO-Gehalt zeigte der Sensor eine geringere Sensorantwort auf Toluol. Mögliche Gründe für dieses Verhalten könnten eine geringere Anzahl von Grenzflächen zwischen F-rGO und Polymer oder eine verminderte Ausbildung des Perkolationsnetzwerks durch Zugabe eines höheren F-rGO-Anteils und der resultierenden Bildung von Aggregaten sein. Die hohe Empfindlichkeit von F-rGO:Polymer 1:5 gegenüber unterschiedlichen Konzentrationen von Toluol bestätigt den zufriedenstellenden $\mathrm{Re}$ duktionsprozess von GO und die homogene Verteilung von F-rGO in der PMMA-Matrix.

Das erhaltene F-rGO/Polymer-Nanokomposit hat sich in dieser Arbeit als vielversprechendes Material für den Nachweis von Toluol etabliert. Es ist davon auszugehen, dass durch die Optimierung der Zusammensetzung und Durchmischung eine weitere Verbesserung des Komposits möglich ist, um bessere elektrische Eigenschaften im Bereich der Sensoranwendung zu erreichen. Darüber hinaus sind systematischere Studien erforderlich, um mehr Einblicke in den Sensormechanismus von F-rGO/Polymer gegenüber vOCs zu gewinnen.

\section{Literatur}

[1] Yuan, W.; Huang, L.; Zhou, Q.; \& Shi, G.: Ultrasensitive and selective nitrogen dioxide sensor based on self-assembled graphene/polymer composite nanofibers. ACS applied materials \& interfaces, 6(19) (2014), S.17003-17008.

[2] Ma, X.; Xie, G.; Su, Y.; Du, H.; Xie, T.; \& Jiang, Y.: Polyvinylpyrrolidone/graphene oxide thin films coated on quartz crystal microbalance electrode for $\mathrm{NH} 3$ detection at room temperature. Science China Technological Sciences, 59(9), (2016), S.1377-1382.

[3] Qi, P.; Zhao, C.; Wang, R.; Fei, T.; \& Zhang, T.: Highperformance QCM humidity sensors using acidizedmultiwalled carbon nanotubes as sensing film. IEEE Sensors Journal, 18(13), (2018), S. 5278-5283.

[4] Basu, S.; \& Bhattacharyya, P.: Recent developments on graphene and graphene oxide based solid-state gas sensors. Sensors and Actuators B: Chemical, 173, (2012), S.1-21.

[5] Thriumani, R.; Zakaria, A.; Omar, M. I.; \& Halim, F. A.: An initial study on oxidized graphene-coated QCM based gas sensor for cancer related volatile sensing application. Recent Innovations in Chemical Engineering (Formerly Recent Patents on Chemical Engineering), 11(1), (2018). S. 29-39.

[6] Kaushik, A.; Kumar, R.; Jayant, R. D.; \& Nair, M.: Nanostructured gas sensors for health care: an overview. Journal of personalized nanomedicine, 1(1), (2015). S.10.

[7] Kauffman, Douglas R., and Alexander Star. "Carbon nanotube gas and vapor sensors." Angewandte Chemie International Edition 47, no. 35 (2008): 65506570.

[8] Salavagione, H.J.; Diez-Pascual, A.M.; Lázaro, E.; Vera, S. and Gomez-Fatou, M.A.: Chemical sensors based on polymer composites with carbon nanotubes and graphene: the role of the polymer. Journal of Materials Chemistry A, 2(35), (2014). S.14289-14328.

[9] Yang, Y.; Li, S.; Yang, W.; Yuan, W.; Xu, J.; \& Jiang, $Y .:$ In situ polymerization deposition of porous conducting polymer on reduced graphene oxide for gas sensor. ACS applied materials \& interfaces, 6(16), (2014). S.13807-13814.

[10] Hidayah, N. M. S.; Liu, W. W. ; Lai, C. W. ; Noriman, N. Z. ; Khe, C. S. ; Hashim, U. ; \& Lee, H. C.: Comparison on graphite, graphene oxide and reduced graphene oxide: Synthesis and characterization. In AIP Conference Proceedings (Vol. 1892, No. 1, p. 150002). AIP Publishing LLC, October 2017

[11] Compton, O. C.; \& Nguyen, S. T.: Graphene oxide, highly reduced graphene oxide, and graphene: versatile building blocks for carbon-based materials. Small, 6(6), (2010). S. 711-723.

[12] Jeffery, A. A.; Rao, S. R.; \& Rajamathi, M.: Preparation of MoS2-reduced graphene oxide ( $\mathrm{GGO}$ ) hybrid paper for catalytic applications by simple exfoliationcostacking. Carbon, 112, (2017). S. 8-16.

[13] Krishnamoorthy, K.; Veerapandian, M.; Yun, K.; \& Kim, S. J.: The chemical and structural analysis of graphene oxide with different degrees of oxidation. Carbon, 53, (2013), S.38-49.

[14] Pham, V. H.; Dang, T. T.; Hur, S. H.; Kim, E. J.; \& Chung, J. S.: Highly conductive poly (methyl methacrylate)(PMMA)-reduced graphene oxide composite prepared by self-assembly of PMMA latex and graphene oxide through electrostatic interaction. ACS applied materials \& interfaces, 4(5), (2012), S. 2630 2636.

[15] Sehrawat, P.; Islam, S. S.; Mishra, P.; \& Ahmad, S.: Reduced graphene oxide ( $r \mathrm{GO}$ ) based wideband optical sensor and the role of Temperature, Defect States and Quantum Efficiency. Scientific reports, 8(1), (2018), S.1-13.

[16] Sheka, E. F.: The uniqueness of physical and chemical natures of graphene: their coherence and conflicts. International Journal of Quantum Chemistry, 114(16), (2014), S. 1079-1095. 
[17] Wang, Z.; Li, P.; Chen, Y.; He, J.; Zhang, W.; Schmidt, O. G.; \& Li, Y.: Pure thiophene-sulfur doped reduced graphene oxide: synthesis, structure, and electrical properties. Nanoscale, 6(13), (2014), S. 7281-7287.

[18] Lee, C.; Wei, X.; Kysar, J. W.; \& Hone, J.: Measurement of the elastic properties and intrinsic strength of monolayer graphene. Science, 321(5887), (2008), S. 385-388.

[19] Krishna, R., Fernandes, D. M.; Venkataramana, E.; Dias, C.; Ventura, J.; Freire, C.; \& Titus, E.: Improved reduction of graphene oxide. Materials Today: Proceedings, 2(1), (2015), S. 423-430.

[20] Joseph, Y.; Guse, B.; Yasuda, A.; \& Vossmeyer, T.: Chemiresistor coatings from Pt-and Au-nanoparticle/nonanedithiol films: sensitivity to gases and solvent vapors. Sensors and Actuators B: Chemical, 98(2-3), (2014), S. 188-195.

[21] Thakur, S.; \& Karak, N.: Green reduction of graphene oxide by aqueous phytoextracts. Carbon, 50(14), (2012) S. 5331-5339.

[22] Tripathi, S. N.; Saini, P.; Gupta, D.; \& Choudhary, V.: Electrical and mechanical properties of PMMA/reduced graphene oxide nanocomposites prepared via in situ polymerization. Journal of materials science, 48(18), (2013). S. 6223-6232.
[23] Wang, C.; Zhang, Y.; Lin, L.; Ding, L.; Li, J.; Lu, R.; ... \& Cheng, R.: Thermal, mechanical, and morphological properties of functionalized graphene-reinforced biobased polyurethane nanocomposites. European Journal of Lipid Science and Technology, 117(12), (2015), S. 1940-1946.

[24] Zhang, Z.; Gao, T.; Si, S.; Liu, Q.; Wu, Y.; \& Zhou, G.: One-pot preparation of $P$ (TA-TEPA)-PAM-RGO ternary composite for high efficient $\mathrm{Cr}(\mathrm{VI})$ removal from aqueous solution. Chemical Engineering Journal, 343, (2018), S. 207-216.

[25] Cao, L., Han, G. C., Xiao, H., Chen, Z., \& Fang, C.: A novel 3D paper-based microfluidic electrochemical glucose biosensor based on rGO-TEPA/PB sensitive film. Analytica chimica acta, 1096, (2020), S. 34-43.

\section{Danksagung}

Wir danken unserem Kollegen Adam Jörg für wertvolle technische Unterstützung und Angela Reichel für die Mithilfe im Chemielabor (IESM). Besonderer Dank geht an Dr. Himcinschi (Institut für Theoretische Physik, TU Bergakademie Freiberg) und Brigitte Bleiber (Institut für Werkstoffwissenschaft, TU Bergakademie Freiberg) für die Durchführung der Raman- beziehungsweise REM-Untersuchungen. 